\title{
Intervirology
}

Abe, H. 209

Aibe, H. 238

Asayama, Y. 238

Awai, K. 125

Choi, B.I. 162

Ebara, M. 277

Han, J.K. 162

Honda, H. 238

Hori, M. 209

Ichikawa, T. 252

Irie, H. 238

Ito, K. 134

Kakihara, D. 238

Kawanaka, K. 125

Kawata, S. 209
Kim, S.H. 162

Kim, T. 209

Kojiro, M. 179

Kondo, F. 277

Koshima, Y. 277

Krinsky, G. 191

Kudo, M. 144, 154, 169, 184, 227

Lee, J.M. 162

Lee, J.Y. 162

Matake, K. 238

Matsui, O. 271

Mitchell, D.G. 134

Mizuguchi, Y. 199

Morishita, S. 125

Moriyama, N. 199
Murakami, T. 209

Muramatsu, Y. 199

Nakamura, H. 209

Nakayama, T. 238

Nishie, A. 238

Ojima, H. 199

Tajima, T. 238

Takayasu, K. 199

Tochio, H. 144, 154

Wen, Y.L. 169

Yamashita, Y. 125

Yoshimitsu, K. 238

Zheng, R.Q. 184

Zhou, P. 154, 169, 184

\section{Subject Index Vol. 47, No. 3-5, 2004}

Abnormal liver circulation 277

Adenomatous hyperplasia 199

Advanced HCC 144

Afferent vessel 144

Anomalous portal tract syndrome 277

Atypical large well-differentiated hepatocellular carcinoma 227

Benign-nature nodule 227

Blood supply 271

Chronic hepatitis 134

Cirrhosis 134, 191

Coded phase inversion harmonic US 169

Color Doppler imaging 144, 154

Combination of CT and angiography 199

Computed tomography (CT) 169, 191, 199, $238,252,271$

Contrast agents 252

- media 162, 169, 184

Dynamic study 209
Dysplastic nodule 144, 191, 199, 271

Early stage HCC 144

Efferent vessel 144

Focal nodular hyperplasia 277

Harmonic study 169

Helical CT 125

Hemangioma focal nodular hyperplasia 154

Hemodynamics 184, 238

Hepatic morphologic change 134

- tumors 154

Hepatocarcinogenesis 271

Hepatocellular carcinoma 144, 154, 169 , 179, 191, 209, 252, 271

- -, early 199

- nodule 238

Human hepatocarcinogenesis 227

Intranodular hemodynamics 227

- portal venous flow 227
Large regenerative nodule 277

Liver neoplasm 125, 162, 169, 184, 252

- transplantation 191

Magnetic resonance (MR) 238, 252

Magnetic resonance imaging (MRI) 125, 134, 191, 209

Maximum velocity 154

Metastatic hepatic cancer 154

Multidetection row CT 209

Nodule-in-nodule 179

Nodular regenerative hyperplasia 277

Portal hypertension 134

Pseudolesion 238

Pulsatility index intratumoral blood flow signal 154

Pulse sequences 252

SPIO 209

Ultrasound 162, 169, 184

\section{KARGER}

(C) 2004 S. Karger AG, Basel

Fax + 41613061234

E-Mail karger@karger.ch

www.karger.com 